\title{
Optimized mixed-domain signal synthesis for broadband impedance spectroscopy measurements on lithium ion cells for automotive applications
}

\author{
Peter Haußmann and Joachim Melbert \\ Forschungsgruppe Kfz-Elektronik, Ruhr Universität Bochum, Universitätsstr. 150, 44801 Bochum, Germany \\ Correspondence to: Peter Haußmann (peter.haussmann@est.rub.de)
}

Received: 24 August 2016 - Revised: 28 December 2016 - Accepted: 3 January 2017 - Published: 1 February 2017

\begin{abstract}
A new impedance spectroscopy measurement procedure for automotive battery cells is presented, which is based on waveform shaping. The method is optimized towards a short measurement duration, high excitation power and increased frequency resolution and overcomes limitations of established methods. For a given spectral magnitude profile, a corresponding time domain waveform is derived from the inverse discrete Fourier transform. Applying an identical initial phase angle for each frequency component, the resulting signal exhibits a high peak-to-peak amplitude at relatively low total excitation power. This limits the maximum allowed power for quasi-linear excitation. Altering the phase angles randomly spreads the excitation power across the complete measurement duration. Thereby, linearity is preserved at higher excitation power. A large set of phase patterns is evaluated statistically in order to obtain a phase pattern with a significant peak-to-peak amplitude decrease. By means of numerical optimization, even further peak-to-peak amplitude reduction is achieved. Including window functions in the synthesis concept minimizes spectral leakage without compromising the spectral signal magnitude in the frequency range of interest. A time domain waveform optimized for impedance spectroscopy on lithium ion cells is synthesized based on the proposed approach and evaluated on real automotive cells. The resulting impedance data show good concordance with established standard measurement procedures at significantly reduced measurement duration and charge throughput. Additionally, increased frequency resolution is achieved, enhancing the level of detail of the obtained impedance data. The method is used for improved localization of aging effects in the cells, without further stress of the cells by the measurement procedure.
\end{abstract}

\section{Introduction}

Lithium ion cells for use in electric vehicles are subject to ongoing research, aiming to reach higher energy densities and longer lifetimes while maintaining safe operation (Banerjee et al., 2017). To reach these goals, the cell chemistry is steadily optimized. To evaluate the performance of a cell technology, benchmark and cycle life, studies are conducted (Fischnaller et al., 2011). These studies rely on precise and reproducible characterization methods to quantify the cell's key properties, like its internal resistance and capacitance, which are strongly influenced by the material composition of the cell (Schmidt et al., 2011). Therefore, analyzing the aging behavior of various material compounds and the localization of their effects in the cell is mandatory.
Electrochemical impedance spectroscopy (EIS) is an established method in the characterization of electrochemical systems. By analyzing measurement data of current and voltage of a lithium ion cell, the frequency-dependent complex impedance $\underline{Z}(j \omega)$ can be calculated (Lohmann et al., 2015b).

The application of EIS during an ongoing long-term aging test on lithium ion cells for automotive energy storage is a challenging task. EIS is usually performed on small cells with low capacity or on dedicated test structures with lowpower test equipment (Schmidt et al., 2011). However, standard automotive lithium ion cells usually have resistance in the $m \Omega$ range and a storage capacity of several tens of ampere hours, thus requiring high current amplitudes (Huang et al., 2016). 
An established test approach is based on sinusoidal excitation with a given amplitude, which ensures a quasi-linear response. The impedance is measured at a discrete number of frequencies, one at a time. This method is robust against influences of noise, as the spectral excitation power is concentrated on a single frequency. However, especially for low frequencies in the $\mathrm{mHz}$ range, long measurement duration is required and high test charge throughput is obtained (Klotz et al., 2011).

In impedance measurements, a dense frequency resolution is desirable. For the single-frequency approach, several periods at each frequency need to be analyzed sequentially. The complete measurement procedure is very time consuming, especially at low frequencies. The measurement requires a duration of several hours. Therefore, time invariance of the device under test (DUT) cannot be guaranteed (Klotz et al., 2011; Lohmann et al., 2015b).

An alternative approach optimized towards short measurement duration is based on pulse excitation. As opposed to the single-frequency approach, the impedance is simultaneously evaluated at multiple frequencies. The frequency domain characteristic results from the time domain pulse shape. Although rectangular pulses can be synthesized on almost any test equipment, their spectra are less useful due to the non-uniform power density. Using sophisticated test equipment capable of arbitrary waveform generation, spectrally optimized pulse shapes like $\operatorname{sinc}(\sin x / x)$ pulses are favorable (Lohmann et al., 2015b). By employing synthetic excitation signals based on defined spectral magnitude profiles, further optimization of the waveform is possible (Guerrero Cervera et al., 2014). However, excitation power is concentrated around the highest peaks in time domain. Considering the long measurement duration required for the analysis of low-frequency EIS, signal energy is still limited by the boundary conditions for linear excitation and time invariance.

Broadband excitation based on band-limited noise signals avoids temporal energy concentration. However, spectral magnitude is randomly distributed across the observed frequency range for practical noise signals. Therefore, suitable excitation is not guaranteed for all frequencies of interest (Møller, 1986).

In this work, a novel excitation signal generation method is presented, aiming to combine the advantageous spectral magnitude properties of pulse signals with widespread time domain characteristics. This is achieved using a waveformshaping approach based on the inverse discrete Fourier transform (IDFT) in the digital signal synthesis (Symons, 2014), extended by the inherent use of window functions (Harris, 1978).

This paper is based on a previous conference publication (Haußmann and Melbert, 2016), which presents a method to significantly reduce the peak-to-peak amplitude of broadband time domain signals by means of phase variation. This extended version features advanced waveform optimization

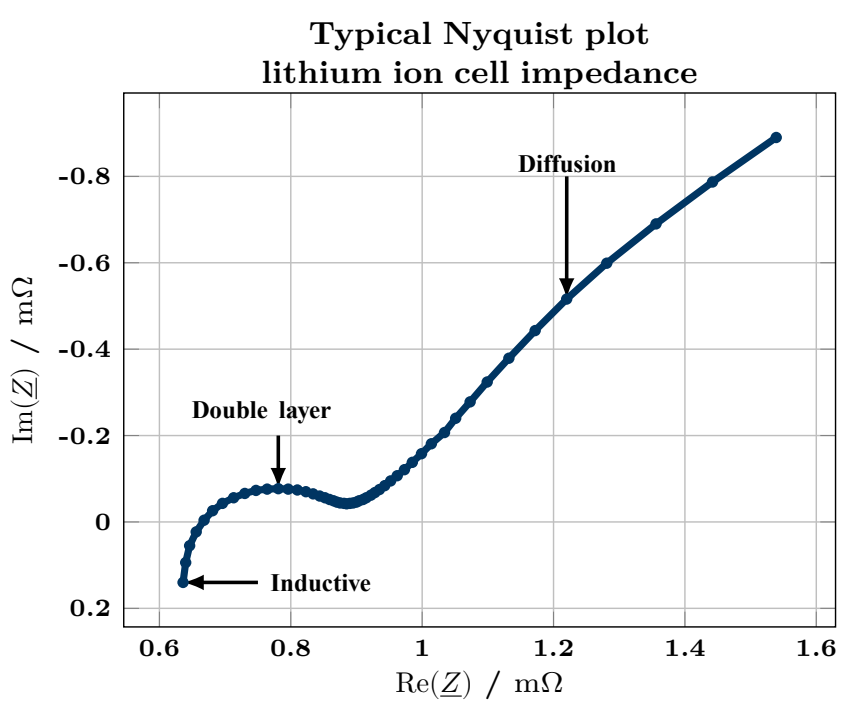

Figure 1. Nyquist plot for lithium ion cell impedance from $10 \mathrm{mHz}$ (top right) to $1 \mathrm{kHz}$ (bottom left). Note the inverse imaginary axis chosen for the reader's convenience.

by combining statistical and numerical techniques in time and frequency domain. The optimization goals are derived from a typical measurement scenario with respect to quality and integrity of impedance spectra. Limited stop-band attenuation and pass-band flatness in the frequency domain are introduced as additional degrees of freedom to achieve an improved optimization result.

\section{Fundamentals}

The impedance of any electrochemical system can be defined as the voltage response following a current excitation (Klotz et al., 2011):

$\underline{Z}(f)=\frac{\underline{V}(f)}{\underline{I}(f)}=|Z| e^{j \Delta \phi}$.

Impedance measurements on a DUT are performed by applying an excitation signal and measuring the system response. In theory, current and voltage excitation can be applied equivalently. In the event of low internal impedance in the $\mathrm{m} \Omega$ range and capacitive behavior, current excitation is more feasible.

For the interpretation of EIS results, the measured impedance is often fitted to an electrochemically motivated equivalent circuit. This allows a link between the measured electrical characteristics and the underlying electrochemical processes inside the cell. The effects observable in automotive lithium ion cells can roughly be separated according to their characteristic frequency range. In the $\mathrm{kHz}$ region, inductive behavior resulting from the cell geometry can typically be identified (Huang et al., 2016). The Hz range is dominated by double-layer capacitance effects at the solid 
electrolyte interphase (SEI) formed between electrodes and electrolyte (Barai et al., 2015). In comparison, lithium ion diffusion processes through the electrolyte are slower by another order of magnitude, determining the impedance in the $\mathrm{mHz}$ range (Schmidt et al., 2011).

In Fig. 1, a typical impedance spectrum of a lithium ion cell is depicted in the Nyquist representation, describing the relation of the real and imaginary part of the impedance.

For robust model fitting, appropriate impedance data quality and integrity are required. Data quality refers to frequency resolution and the signal-to-noise ratio (SNR), which is limited by the excitation signal amplitude and the performance of the test equipment.

Furthermore, the model fitting approach implicitly requires that the underlying system has to be linear and time invariant. These conditions are not necessarily satisfied by a lithium ion cell. Therefore, verification of the EIS results' integrity is required.

The Kramers-Kronig relations describe the interdependency of the real and imaginary part of causal LTI (linear time invariant) system transfer functions in system theory. A Kramers-Kronig compliance test method applicable to EIS results is presented in Schönleber and Ivers-Tiffée (2015). The algorithm is employed to validate the measurement results in this work.

As the impedance is defined in the frequency domain, spectra calculation usually involves a time-frequency domain transform like the discrete Fourier transform (DFT). The inverse DFT (IDFT) is suitable for the synthesis of waveforms featuring well-defined frequency domain properties. The main characteristics of the (I)DFT are briefly summarized in this section.

\section{Time-frequency relations}

For an input time domain signal consisting of $k$ samples at the sampling frequency $f_{\mathrm{S}}$, the minimum frequency $f_{\text {min }}$ considered by the DFT equals the reciprocal value of the total sampling duration $T_{\text {Meas }}$.

$f_{\text {min }}=\frac{f_{\mathrm{S}}}{k}=\frac{1}{T_{\text {Meas }}}$

According to the well-known Nyquist theorem, the upper frequency bound is calculated based on Symons (2014):

$f_{\max }=\frac{f_{\mathrm{S}}}{2}$.

Therefore, in order to investigate the DUT's impedance at frequency $f_{i}$, the following constraints have to be met:

$$
\begin{aligned}
& f_{\mathrm{S}} \geq 2 f_{i}, \\
& T_{\text {meas }} \geq \frac{1}{f_{i}} .
\end{aligned}
$$

The required sampling frequency range derived from Eq. (4) is not an issue in state-of-the-art measurement systems. However, in the $\mathrm{mHz}$ range, the constraint in Eq. (5) regarding $T_{\text {Meas }}$ might lead to extremely long measurement durations.

In theory, $f_{\min }=\left(T_{\text {meas }}\right)^{-1}$ is the minimum frequency analyzed by the DFT. However, signal components containing a frequency of lower than $f_{\min }$ contribute to the low-frequency range as undesired artifacts of the DFT spectrum due to spectral leakage. To avoid measurement errors in practical scenarios, the number of periods $N$ analyzed per frequency is usually chosen as a multiple of the maximum period length of interest, resulting in even longer measurement durations. In typical measurement scenarios, $N \geq 3$ is an adequate choice.

2. Properties of real valued time domain signals

The IDFT of a frequency domain signal $\underline{X}(f)$ yields a complex valued time domain representation $\underline{x}(t)$ in general. Imaginary signal components of $\underline{x}(t)$, which cannot be interpreted meaningfully for time domain signals, can be avoided by considering symmetry properties of the Fourier transform. Every real valued time domain signal exhibits a complex conjugate spectrum, with even absolute values and odd phase angles as noted in Eqs. (6) and (7) (Sundararajan, 2001).

$$
\begin{aligned}
& |\underline{X}(f)|=|\underline{X}(-f)| \\
& \phi(f)=-\phi(-f)
\end{aligned}
$$

In this work, the IDFT is performed on complex conjugate frequency domain signals only. As a consequence, the resulting time domain waveforms are purely real valued and can therefore be fully synthesized using an arbitrary waveform generator.

\section{Signal power}

The power $P(f)$ of a frequency domain signal is proportional to its magnitude squared, as noted in Eq. (8) (Lyons, 2011).

$$
P(f) \sim|\underline{X}(f)|^{2}
$$

Therefore, for power-sensitive applications like impedance spectroscopy, even a minor magnitude raise is significantly beneficial.

\section{Impedance spectroscopy measurement scenario}

An overview of a typical EIS measurement scenario is depicted in Fig. 2. In order to analyze the impedance of DUT, adequate excitation in the frequency range of interest is required. This can be accomplished using various types of signals. The selected waveform is generated by an excitation current source to stimulate the DUT. The excitation and the 


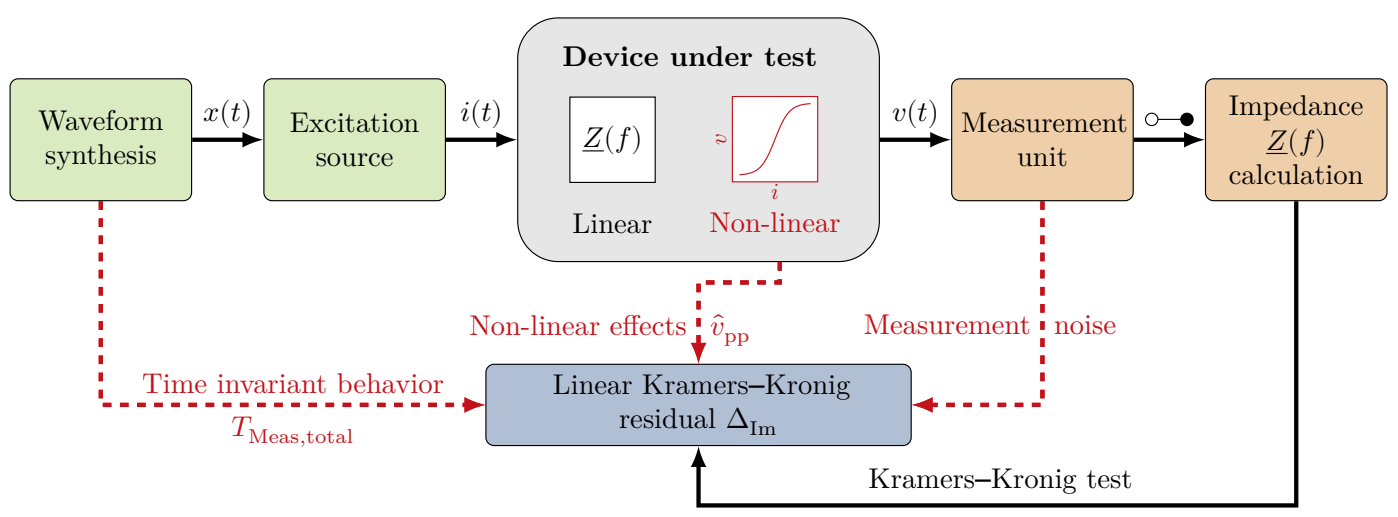

Figure 2. Flow chart of an impedance spectroscopy measurement scenario. The solid arrows describe a generic EIS measurement procedure, while the dashed red arrows indicate typical error contributions.

response signal are recorded simultaneously in the time domain by a measurement unit. With appropriate digital postprocessing, including the DFT, the DUT's impedance can be calculated.

Noise effects induced by non-ideal components in the measurement unit compromise the resulting data quality. Besides noise influence, additional measurement errors not related to noise may occur, because the DUT's impedance is interpreted as the transfer function of a causal, linear and timeinvariant system for most applications. Only if these conditions are satisfied, the EIS results can be considered valid. The aforementioned linear Kramers-Kronig test (LinKK) is a suitable method to verify data integrity (Schönleber and Ivers-Tiffée, 2015). The imaginary part of the impedance is calculated for the measured real part of the spectrum with respect to the Kramers-Kronig conditions. The deviation of the measured and calculated imaginary parts is referred to as the linear Kramers-Kronig residual $\Delta_{\mathrm{Im}}$.

\subsection{Residual contributions}

In an ideal measurement scenario, the mean residual $\overline{\Delta_{\mathrm{Im}}}$ is equal to zero. However, for practical EIS studies, various error influences have to be considered. The major residual contributors and feasible counter measures are discussed in the following.

1. Time-variant behavior due to variation of the cell's state of charge or temperature

The impedance of a lithium ion cell is dependent on various influence factors, such as temperature, state of charge and state of health. Therefore, the impedance changes over time $\left(\underline{Z}=f\left(f, t_{0}\right)\right)$. However, in EIS measurements, a time-invariant impedance $\underline{Z}(f)$ is measured, observing only the actual cell state, which is assumed to be constant during the entire measurement duration. This assumption is not necessarily valid. To avoid impedance errors related to time-invariant system behavior, sufficiently long rest times are required before the EIS measurement starts. Time-consuming measurement procedures are prone to errors due to impedance drift during the measurement, especially if the DUT's temperature or state of charge is influenced by the measurement procedure itself. This asks for time-efficient EIS procedures with low energy and charge throughput (Klotz et al., 2011).

2. Linearity violation caused by excessive peak-to-peak voltage amplitudes

Non-linear effects in the DUT are stimulated by high values of the peak-to-peak voltage amplitude $\hat{v}_{\mathrm{pp}}$ during the measurement. This leads to signal distortion, resulting in a redistribution of the signal power in the response signal's frequency spectrum. This phenomenon cannot be described by the a priori linear impedance $\underline{Z}(f)$. To avoid errors due to non-linear behavior, the peak-to-peak excitation amplitude $\hat{x}_{\mathrm{pp}}$ must be limited (Lohmann et al., 2015b).

\section{Noise contributions}

Noise influences induced by non-ideal components of the measurement unit also lead to increased values of the residual $\Delta_{\mathrm{Im}}$. In this case, the residual error is homogeneously distributed across the entire frequency range (Schönleber and Ivers-Tiffée, 2015). In contrast to the effects discussed above, noise contributions do not violate the Kramers-Kronig conditions. Yet it is difficult to differentiate between actual violations against the Kramers-Kronig constraints and noise-induced effects. Noise contributions can be minimized by increasing the excitation power, as an improved signal-to-noise ratio is reached.

Obviously, these constraints are in contradiction to each other, as the spectral magnitude $|\underline{X}(f)|$ influences the time domain peak-to-peak amplitude $\hat{x}_{\mathrm{pp}}$ to some extent. In Fig. 3 , the impact of the peak-to-peak voltage amplitude $\hat{v}_{\mathrm{pp}}$ on 


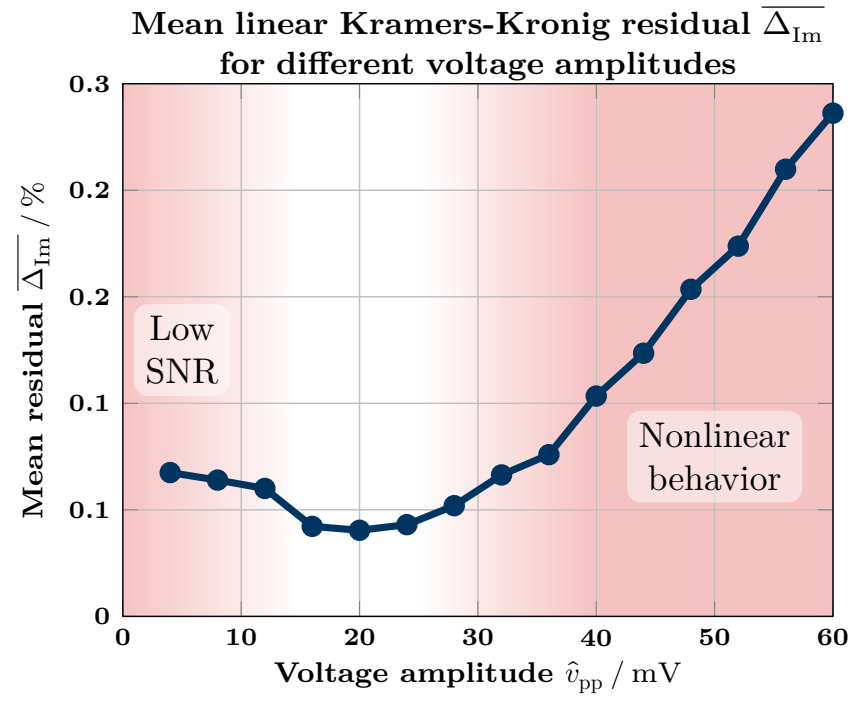

Figure 3. Arithmetic mean of the linear Kramers-Kronig residual $\overline{\Delta_{\text {Im }}}$ for different voltage amplitudes in stepped-sine EIS measurements on an automotive lithium ion cell (Lohmann et al., 2015b). The red color shading indicates the amplitude values unsuitable for EIS measurements due to linearity violations $\left(\hat{v}_{\mathrm{pp}}>25 \mathrm{mV}\right)$ and noise contributions $\left(\hat{v}_{\mathrm{pp}}<15 \mathrm{mV}\right)$.

the Kramers-Kronig residual is depicted. The underlying investigation uses controlled current excitation to achieve the specified voltage amplitude $\hat{v}_{\text {pp }}$ (Lohmann et al., 2015b). Increased residual values occur due to noise contributions at amplitudes below $15 \mathrm{mV}$. For amplitudes exceeding $25 \mathrm{mV}$, a significant residual increase caused by non-linear effects is observed. Therefore, the peak-to-peak voltage excitation should be chosen as $\hat{v}_{\mathrm{pp}} \approx 20 \mathrm{mV}$ to reduce the impact of measurement noise while maintaining linear excitation.

The excitation source and the measurement unit are encapsulated into an electrochemical workstation and cannot be replaced by alternative components. As a consequence, noise performance improvements achieved by hardware modification are generally not available. Instead, this work focuses on defining an optimized measurement procedure based on improved waveform shapes to enhance the data quality without compromising data integrity, regardless of the test equipment used.

\section{Standard impedance spectroscopy measurement procedures}

This section provides a short overview of established EIS measurement procedures, discussing the main advantages and disadvantages of each method.

In a conventional EIS approach, the DUT is stimulated at only one frequency at a time using sinusoidal excitation signals. In literature, this procedure is referred to as stepped-sine EIS (Lohmann et al., 2015a). The entire excitation power is concentrated on a single frequency, resulting in an excellent signal-to-noise ratio.

The complete measurement duration can be calculated using the following equation, assuming $N$ periods of $M$ different frequencies.

$T_{\text {Meas, total }, \sin }=\sum_{i=1}^{M} \frac{N}{f_{i}}$

In the $\mathrm{mHz}$ range, signal periods last up to more than $15 \mathrm{~min}$. Depending on the desired frequency range and resolution, typical values of $T_{\text {Meas,total,sin }}$ range from several hours up to days. Time invariance cannot be assumed during such long measurement durations. Additionally, the time frame acceptable for EIS measurements in cycle life studies is usually limited, which requires time-efficient EIS methods.

In order to reduce measurement duration while preserving the same frequency resolution, broadband excitation using pulse waveforms can be applied. As opposed to sinusoidal signals, multiple frequency components are present in the excitation signal (Klotz et al., 2011).

The spectral density characteristic determine the performance of a pulse waveform for EIS. For most commercial test equipment, only rectangular pulses are available, suitable for measurements with low demand on duration and charge throughput. Such an excitation waveform provides a non-uniform power density across the frequency range of interest, which results in a poor signal-to-noise ratio.

Alternative pulse forms like sinc pulses enable a homogeneous broadband excitation (Lohmann et al., 2015b). The sinc pulse in time domain is defined in Eq. (10).

$x_{\text {sinc }}(t)=\frac{\sin (2 \pi f t)}{2 \pi f t}$

Equivalently, the time domain waveform of the sinc pulse can be interpreted as the result of the inverse Fourier transform of a rectangular spectral magnitude profile (Land et al., 2007).

For pulse signals covering the complete frequency range of interest, the required measurement duration is calculated as a multiple of the period of the minimum frequency of interest:

$T_{\text {Meas, total, pulse }}=\frac{N}{f_{\min }}$.

However, the spectral excitation power is shared by all frequency components of the stimulus signal, offering a limited signal-to-noise ratio with respect to each frequency component. This can be overcome by using multiple excitation pulses covering different frequency ranges. For a compromise between the single-frequency approach and a fullbandwidth broadband profile, a relative bandwidth of one frequency decade achieves reasonably short measurement durations and adequate excitation power in the frequency range of interest. In this example, the overall measurement dura- 
tion for a desired bandwidth of $L$ decades results in

$T_{\text {Meas,total,pulse }}=\sum_{k=1}^{L} \frac{N}{10^{k-1} \cdot f_{\min }} \approx 1.1 \frac{N}{f_{\min }}$.

A comparison between Eqs. (9) and (12) shows a significant reduction of measurement duration for the pulse EIS approach compared to stepped-sine procedures.

Even spectrally optimized pulse shapes like sinc pulses show low average signal power at high peak-to-peak amplitude in time domain, limiting the achievable quality of the measured impedance spectra due to linearity constraints.

As an alternative to broadband pulses, signals with more than one discrete frequency component can be used to simultaneously provide sparse broadband excitation, which is also referred to as multi-sine excitation (Gamry Instruments, 2011). Based on the choice of frequency components, significant reduction of the measurement duration compared to stepped-sine measurements can be achieved. However, the selection of an appropriate frequency distribution requires a priori knowledge about the system under investigation (Sanchez et al., 2011; Koch and Jossen, 2014).

In the following, a generic signal synthesis concept for broadband signals is presented, which does not require a priori information about the DUT.

\section{Time domain waveform synthesis for arbitrary spectral magnitude profiles}

In this section, a waveform-shaping method is developed. The design flow can be used to synthesize time domain waveforms based on an arbitrary spectral magnitude profile $|\underline{X}(f)|$, taking the constraints identified in Sect. 3.1 into account.

The measurement duration cannot be reduced significantly below $T_{\text {Meas,total,pulse }}$ specified in Eq. (12), as the value is chosen for a given frequency range with respect to the fundamental properties of the (I)DFT described in Sect. 2. A signal synthesis work flow dedicated to synthesize signals with the lowest achievable peak-to-peak amplitude $\hat{x}_{\mathrm{pp}}$ is defined, maintaining the spectral magnitude characteristics $|\underline{X}(f)|$.

\subsection{Basic approach}

The time domain waveform $x_{0}(t)$ is synthesized by applying the IDFT to the spectral profile $\underline{X}(f)$, which is defined by its magnitude $|\underline{X}(f)|$. The frequency sampling points $f_{i}$ of the profile are dependent on the total signal duration $T_{\text {sig }}$ and the sampling frequency $f_{\mathrm{S}}$, in accordance with the aforementioned properties of the (I)DFT. For each frequency component $f_{i}$, the target magnitude $\left|\underline{X}\left(f_{i}\right)\right|$ is defined.

$\left|\underline{X}\left(f_{i}\right)\right|=\left\{\begin{array}{l}1, \text { if } f_{\min } \leq f_{i} \leq f_{\max } \\ 0, \text { otherwise }\end{array}\right.$
The chosen target frequency domain excitation profile defined in Eq. (13) exhibits a rectangular shape with homogeneous density from $f_{\min }$ up to $f_{\max }$. As noted in Eqs. (14) and (15), a relative bandwidth of one frequency decade is covered, including a $10 \%$ margin above $f_{\max }$.

$$
\begin{aligned}
f_{\text {min }} & =\frac{f_{\mathrm{S}}}{100} \\
f_{\max } & =1.1 \frac{f_{\mathrm{S}}}{10}
\end{aligned}
$$

The signal duration is chosen to $T_{\text {sig }}=4 \cdot\left(f_{\min }\right)^{-1}$ in order to avoid errors due to low-frequency artifacts. The sampling frequency $f_{\mathrm{S}}$ may be chosen with respect to the frequency range of interest. The total EIS measurement duration is calculated according to Eq. (12) in analogy to methods based on established pulse waveforms (Klotz et al., 2011).

For the unambiguous definition of frequency domain signals, both magnitude information $\left|\underline{X}\left(f_{i}\right)\right|$ and the initial phase angle $\phi_{i}$ are required. In the first approach, the phase angle is chosen as

$\phi_{i}=0 \forall i$.

The resulting time domain waveform $x_{0}(t)$ is depicted in Fig. 4 and resembles a frequency-modulated sinc pulse. It shows high temporal concentration of the signal power near the center of the waveform, as expected in analogy to common pulse synthesis approaches. This leads to a high peak-topeak amplitude $\hat{x}_{\mathrm{pp}}$. As a consequence, the excitation power available in EIS measurements is limited due to linearity constraints.

\subsection{Peak-to-peak amplitude reduction by means of phase variation}

As mentioned in Sect. 3.1, high spectral power density and low peak-to-peak amplitude are mandatory in EIS measurements. These constraints are not sufficiently met by the signal $x_{0}(t)$. In the following, a technique to reduce the time domain peak-to-peak amplitude $\hat{x}_{\mathrm{pp}}$ without changing the spectral power density is presented. For sinusoidal signals, the amplitude in time domain and the magnitude in frequency domain are equivalent. In contrast, a large variety of broadband time domain waveforms with different peak-to-peak amplitudes may share equal magnitude characteristics in the frequency domain, distinguishable by the distribution of the phase angle $\phi_{i}$ of each frequency component (Guillaume et al., 2001).

As stated in Eq. (1), for measuring the impedance $\underline{Z}(f)$, only the relative phase angle $\Delta_{\phi}$ between voltage and current is relevant. The absolute initial phase angle $\phi_{i}$ of each excitation frequency component may be altered arbitrarily.

Suitable phase patterns can be employed in order to reduce the peak-to-peak amplitude $\hat{x}_{\mathrm{pp}}$ without altering the spectral magnitude characteristics $|\underline{X}(f)|$. A significant peak-to-peak amplitude reduction can be achieved by applying random 


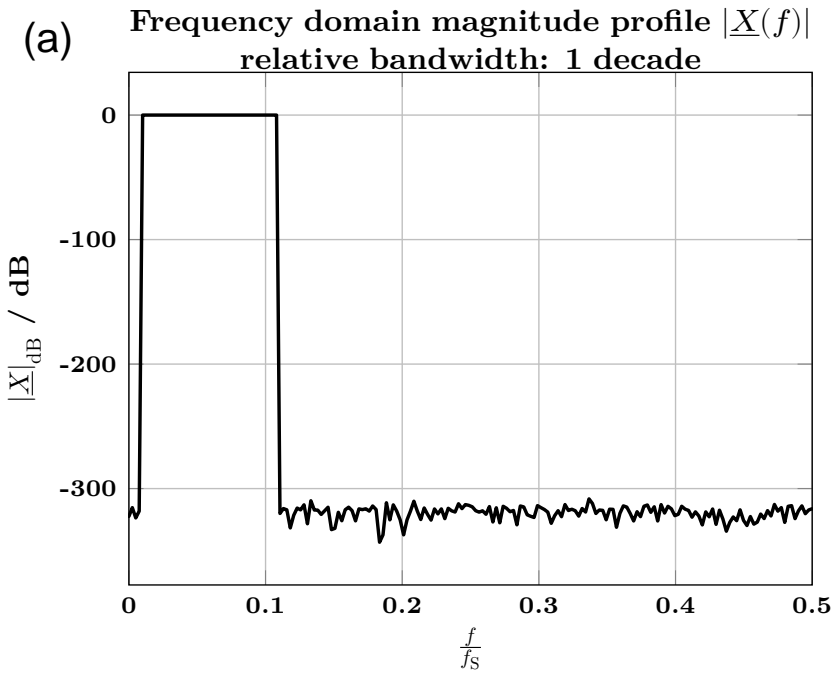

(b)

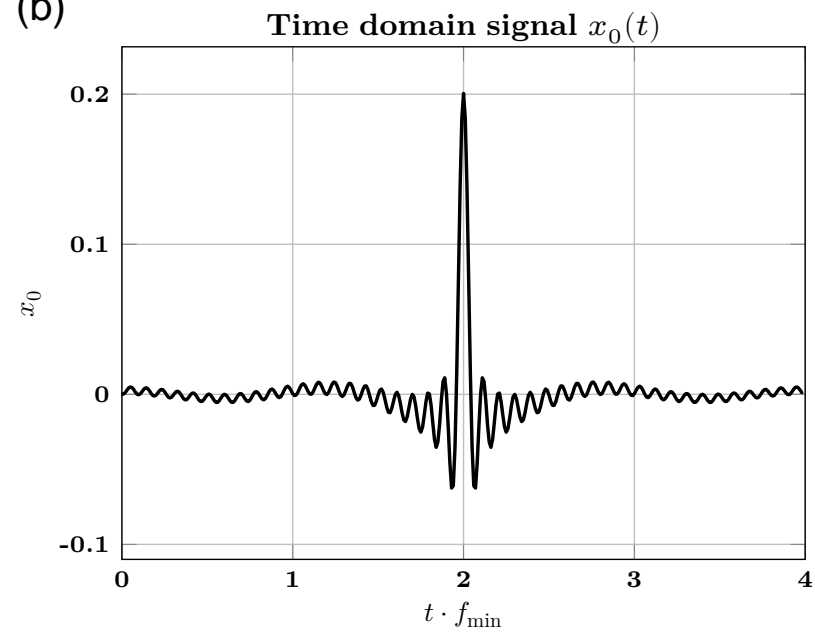

Figure 4. Top: normalized frequency domain magnitude profile for a relative bandwidth of one frequency decade. Bottom: synthesized time domain waveform $x_{0}(t)$.

phase patterns $\phi_{i}(f)$, defined in Eq. (17).

$\phi_{i}=\operatorname{RANDOM}(0: 2 \pi)$

For each frequency component $f_{i}$, any value of $\phi_{i}\left(f_{i}\right)$ ranging from 0 to $2 \pi$ can be chosen. This results in an infinite set of available phase patterns. However, for every phase pattern, the peak-to-peak amplitude is reduced to a different extent. This phenomenon is visualized in Fig. 5 by comparing time domain waveforms with three different random phase patterns to the unmodified signal $x_{0}$ described above. For the given signals, the peak-to-peak amplitude decrease differs significantly. To identify a phase pattern with a high amplitude decrease, a statistical approach is pursued. In this work, 10000 different random phase patterns are evaluated. The distribution of the achievable peak-to-peak amplitude decrease referred to $\hat{x}_{0, \mathrm{pp}}$ is depicted in Fig. 6 . For the chosen patterns, the reduction ranges from 28 to $65 \%$.
In Fig. 8a, the waveform $x_{1}(t)$ is depicted. It is based on the random phase pattern which yields the waveform with the highest peak-to-peak amplitude reduction derived from the statistical analysis. This signal $x_{1}(t)$ is chosen as the initial point for further optimization.

\subsection{Spectral leakage reduction using window functions}

As a consequence of the random phase shift introduced in Sect. 5.2, the resulting time domain signal $x_{1}(t)$ generally does not start and end at zero. Therefore, the waveform is prone to spectral leakage, causing amplitude and phase errors during measurement. This can be avoided by multiplying the signal by a suitable window function $h_{\text {win }}(t)$, which is usually performed in the post-measurement digital signal processing. Thereby, a part of the measured data is discarded and does not contribute to the EIS results. Alternatively, the window function can already be in the excitation (Harris, 1978). In this case, none of the measured information is neglected during the evaluation in this case.

$x_{2}(t)=x_{1}(t) \cdot h_{\mathrm{win}}(t)$

A window function matching the demands of the EIS application must be chosen. Broadband window functions feature a narrow time domain shape, which limits the achievable peak-to-peak amplitude decrease. Therefore, a narrow-band Gaussian window function is used, offering a good compromise between spectral leakage avoidance and low peak-topeak amplitudes (Harris, 1978).

The signal power of $x_{1}(t)$ is localized randomly in time domain. The window function attenuates the signal amplitude for each frequency component to a different extent. As a consequence, the spectral magnitude $\left|\underline{X}_{2}(f)\right|$ of the windowed waveform $x_{2}(t)$ substantially differs from the target magnitude profile $|\underline{X}(f)|$, as depicted in Fig. 8d. The frequencydependent pass-band attenuation of $\left|X_{2}(f)\right|$ can reach to up to $7 \mathrm{~dB}$, which significantly reduces the SNR at several frequencies.

\subsection{Mixed-domain waveform optimization}

Based on the definition in Eq. (13), the spectral magnitude of the synthetic waveforms exhibits a perfectly flat shape in the frequency range of interest as well as infinite stop-band attenuation. In practical measurement scenarios, also finite magnitude flatness can be tolerated without compromising the broadband excitation characteristics. Furthermore, minor signal power contributions in the stop band do not significantly alter the excitation profile. Allowing spectral magnitude shapes with limited flatness and stop-band attenuation allows a new degree of freedom for reducing of the peak-topeak amplitude $\hat{x}_{\mathrm{pp}}$ even further.

A numerical optimization procedure is applied for this purpose. The window function introduced in Sect. 5.3 is 


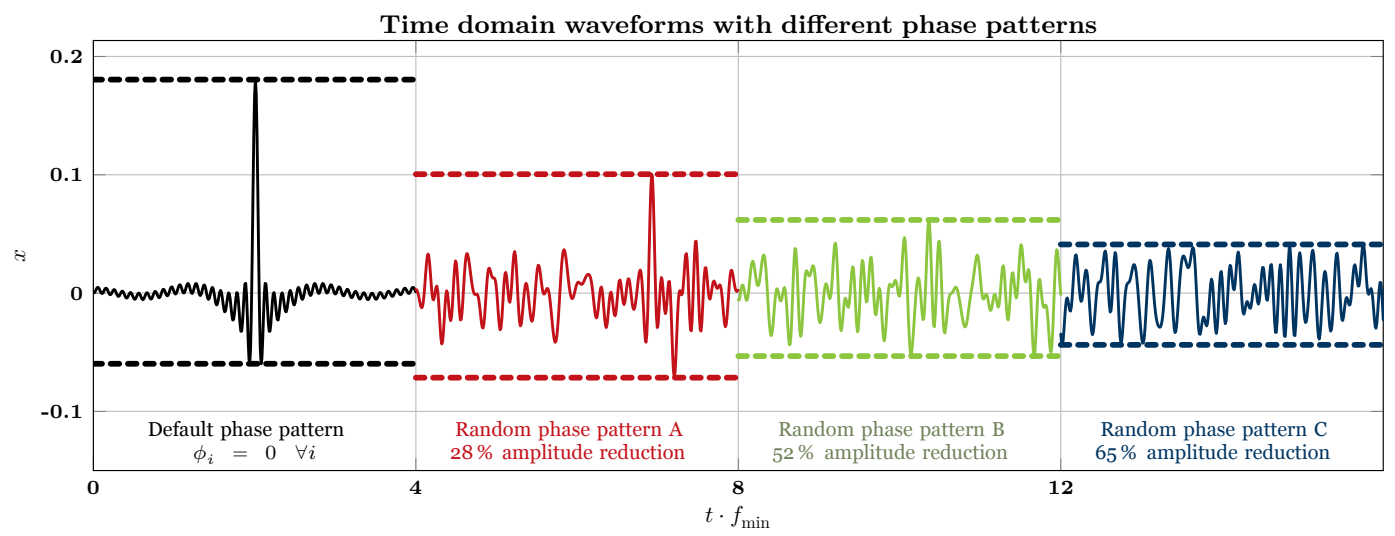

Figure 5. Time domain waveforms with equal spectral magnitude and different phase characteristics.

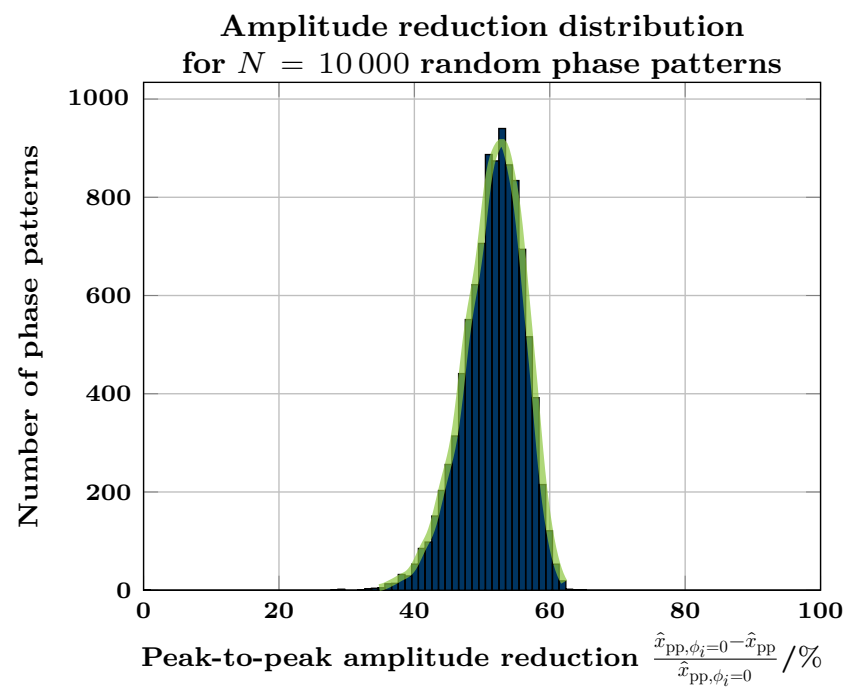

Figure 6. Peak-to-peak amplitude decrease distribution for 10000 random phase patterns

incorporated into the optimization flow, yielding time domain waveforms which are robust against spectral leakage. A flow diagram describing the optimization steps is depicted in Fig. 7.

The optimization uses the well-known LevenbergMarquardt algorithm (Hanke-Bourgeois, 2006). As an initial signal $x(t)$, the waveform $x_{1}(t)$ described in Sect. 5.2 is chosen, as its peak-to-peak amplitude is already conveniently low. A time domain modification function $a(t)$, which is altered by the Levenberg-Marquardt algorithm in every iteration, is added to the signal $x(t)$. Afterwards, the resulting intermediate waveform $x_{\mathrm{A}}(t)$ is multiplied by the window function introduced in Sect. 5.3, yielding the signal $x_{\mathrm{B}}(t)$. These steps are performed repeatedly to minimize the value of the employed cost function $J$, which is defined in Eq. (19).

$$
J=\left(\hat{x}_{\mathrm{pp}}\right)^{w_{1}} \cdot\left(w_{2} \cdot J_{\Delta\left|X_{\mathrm{p}}\right|}+w_{3} \cdot J_{\left|X_{\mathrm{s}}\right|}\right)
$$

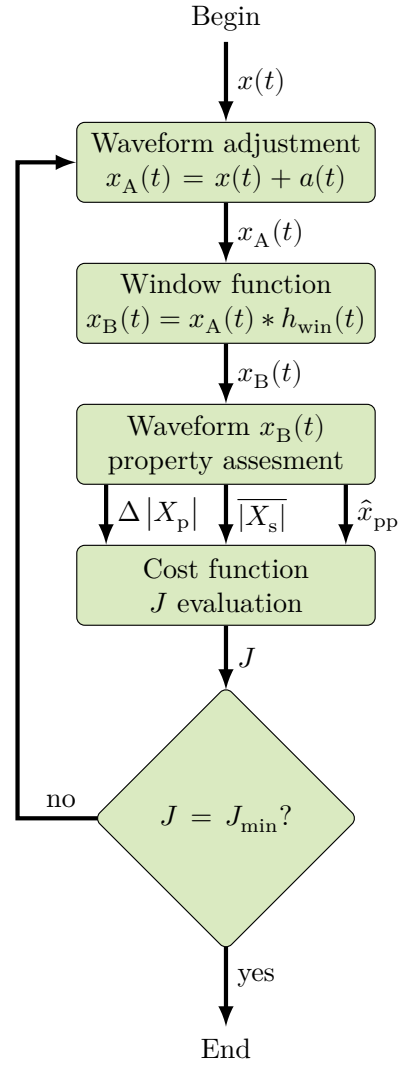

Figure 7. Numerical optimization flow chart.

The cost function $J$ considers the peak-to-peak amplitude $\hat{x}_{\mathrm{pp}}$, the spectral pass-band flatness $\Delta\left|X_{\mathrm{p}}\right|$ and the arithmetic mean of the stop-band attenuation $\left|X_{\mathrm{S}}\right|$. The weighting factors $w_{i}$ can be chosen to emphasize the influence of the associated waveform parameter.

The subsidiary cost functions $J_{\Delta\left|X_{\mathrm{p}}\right|}$ and $J_{\left|X_{\mathrm{s}}\right|}$ are defined in Eqs. (20) and (21), respectively, featuring the threshold 
Table 1. Optimization parameters used for the synthesis of the time domain signal $x_{3}(t)$. Note the emphasis on $\hat{x}_{\mathrm{pp}}$ to set focus on peakto-peak amplitude reduction.

\begin{tabular}{llllll}
\hline Parameter & $\Delta\left|X_{\mathrm{p}}\right|_{\text {th }}$ & $\left|X_{\mathrm{s}}\right|_{\text {th }}$ & $w_{1}$ & $w_{2}$ & $w_{3}$ \\
\hline Value & $\pm 0.1 \mathrm{~dB}$ & $60 \mathrm{~dB}$ & 1.5 & 1 & 0.25 \\
\hline
\end{tabular}

values $\Delta\left|X_{\mathrm{p}}\right|_{\mathrm{th}}$ and $\left|X_{\mathrm{s}}\right|_{\mathrm{th}}$.

$J_{\Delta\left|X_{\mathrm{p}}\right|}= \begin{cases}1, & \text { if } \Delta\left|X_{\mathrm{p}}\right|<\Delta\left|X_{\mathrm{p}}\right|_{\text {th }} \\ 1+\frac{\Delta\left|X_{\mathrm{p}}\right|_{\mathrm{dB}}-\Delta\left|X_{\mathrm{p}}\right|_{\mathrm{th}, \mathrm{dB}}}{20 \mathrm{~dB}}, & \text { else, }\end{cases}$

$J_{\left|X_{\mathrm{s}}\right|}= \begin{cases}1, & \text { if } \overline{\left|X_{\mathrm{s}}\right|}<\left|X_{\mathrm{s}}\right|_{\text {th }} \\ 1+\frac{\left.\overline{\mid X}_{\mathrm{s}}\right|_{\mathrm{dB}}}{\left|X_{\mathrm{s}}\right|_{\text {tht,dB }}}, & \text { else }\end{cases}$

The optimization parameters listed in Table 1 are employed. The parameters were chosen in order to assign the highest priority to the peak-to-peak amplitude reduction, at the cost of moderate pass-band flatness and possibly limited stopband attenuation.

The resulting waveform $x_{3}(t)$ is depicted in Fig. 8c. Compared to the previously discussed signals $x_{1}(t)$ and $x_{2}(t)$, the peak-to-peak amplitude is further reduced. The frequency domain comparison shows that the spectral magnitude characteristics in the pass band closely resemble the initial magnitude profile $|\underline{X}(f)|$ with a pass-band ripple of $\pm 1 \mathrm{~dB}$. More than $95 \%$ of the signal power is located in the frequency range of interest.

\subsection{Waveform synthesis summary}

In the previous sections, a waveform-shaping flow is developed and improved in three steps, each addressing the main disadvantages of the previous version. The signal $x_{0}(t)$ exhibits characteristics similar to a sinc pulse and is chosen as an initial waveform.

By employing random phase patterns, a significant peakto-peak amplitude decrease of up to $66 \%$ is achieved resulting into $x_{1}(t)$, which is selected for exhibiting the lowest peak-to-peak amplitude of all signals based on a large set of phase patterns.

However, $x_{1}(t)$ is not feasible as an EIS excitation waveform due to spectral leakage in real measurement scenarios. As a counter measure, the waveform $x_{2}(t)$ is introduced by applying a window function to $x_{1}(t)$. While substantially reducing the effects of spectral leakage, the target magnitude profile is not satisfied by $\left|X_{2}(f)\right|$, as the combination of the random phase pattern and the window function multiplication causes frequency-dependent attenuation of the spectral signal magnitude. In order to combine the improvements achievable by using random phase patterns and window functions, as well as spectral magnitude boundary conditions in the pass and stop bandwidth, a numerical optimization
Table 2. Comparison of all waveforms discussed in this work.

\begin{tabular}{lrrrrr}
\hline Signal & $x_{0}$ & $x_{1}$ & $x_{2}$ & $x_{3}$ & $x_{3 \mathrm{~b}}^{a}$ \\
\hline Frequency range & \multicolumn{5}{c}{ One frequency decade } \\
Phase variation & & $\checkmark$ & $\checkmark$ & $\checkmark$ & $\checkmark$ \\
Statistical evaluation & & $\checkmark$ & $\checkmark$ & $\checkmark$ & \\
Window function & & & $\checkmark$ & $\checkmark$ & $\checkmark$ \\
Amplitude scaling & & & & $\checkmark$ & $\checkmark$ \\
Numeric optimization & & & & $\checkmark$ & \\
$\hat{x}_{\text {pp }}$ reduction $\frac{\hat{x}_{0, \mathrm{pp}}-\hat{x}_{\mathrm{pp}} / \%}{\hat{x}_{0, \mathrm{pp}}} / X_{\mathrm{p}} \mid / \mathrm{dB}$ & 0 & 0 & 67 & 71 & 60 \\
Magnitude flatness $\Delta \mid$ & \pm 1 & 0 \\
Pass-band power increase/dB & 0 & 9.3 & 9.6 & 10.6 & 8.0 \\
\hline * The signal $x_{3 \mathrm{~b}}$ corresponds to the signal $x_{\text {ISW }}^{\prime}$ presented in Haußmann and Melbert \\
(2016).
\end{tabular}

scheme is defined. This results into waveforms with the lowest peak-to-peak amplitude. The final waveform $x_{3}(t)$ provides the best trade-off for the requirements in time and frequency domain and is used for all measurements in this work.

A comparison of all signal optimization stages presented in this work is given in Table 2. The table also includes the signal $x_{3 \mathrm{~b}}$, which corresponds to the waveform $x_{\mathrm{ISW}}^{\prime}$ (intrasynthesis window function) presented in the previous version of this work (Haußmann and Melbert, 2016). This signal is based on the same frequency domain magnitude profile as used in this work, but exhibits a different phase pattern, which has been chosen arbitrarily in contrast to the choice of the phase pattern achieving the most significant amplitude decrease proposed in this work. The signal $x_{3 \mathrm{~b}}$ takes advantage of a numeric rescaling procedure, which compensates the amplitude variation introduced by the window function multiplication, which is observed for the signal $x_{2}$. However, the advanced constraints regarding pass-band flatness and stop-band attenuation introduced in Sect. 5.4 are not considered for $x_{3 \mathrm{~b}}$. Altogether, only a $60 \%$ amplitude decrease is achieved for $x_{3 \mathrm{~b}}$, while the amplitude of the statistically and numerically optimized signal $x_{3}$ is reduced by $71 \%$.

\section{Measurement results}

For the practical evaluation of the proposed measurement procedure, a precision lithium ion single-cell test unit developed by our research group was used (Lohmann et al., 2015 b). It provides a wide bandwidth linear $600 \mathrm{~A}$ power stage along with a measurement control unit, enabling accurate measurements with errors below $0.1 \%$ (Weßkamp et al., 2016). Furthermore, arbitrary current profiles can be generated at sampling rates up to $10 \mathrm{kHz}$. The DUT is a single automotive cell for the use in plug-in hybrid vehicles (PHEV) with a nominal capacity in the range of about $30 \mathrm{Ah}$. All measurements are conducted at $25^{\circ} \mathrm{C}$ ambient temperature and a $50 \%$ state of charge.

In order to verify the spectral characteristics of the proposed waveform, $x_{3}(t)$ is applied to the DUT as a current profile, defined in Eq. (22). The current amplitude $\hat{i}_{\text {pp }}$ has 
(a) IDFT of rectangular magnitude
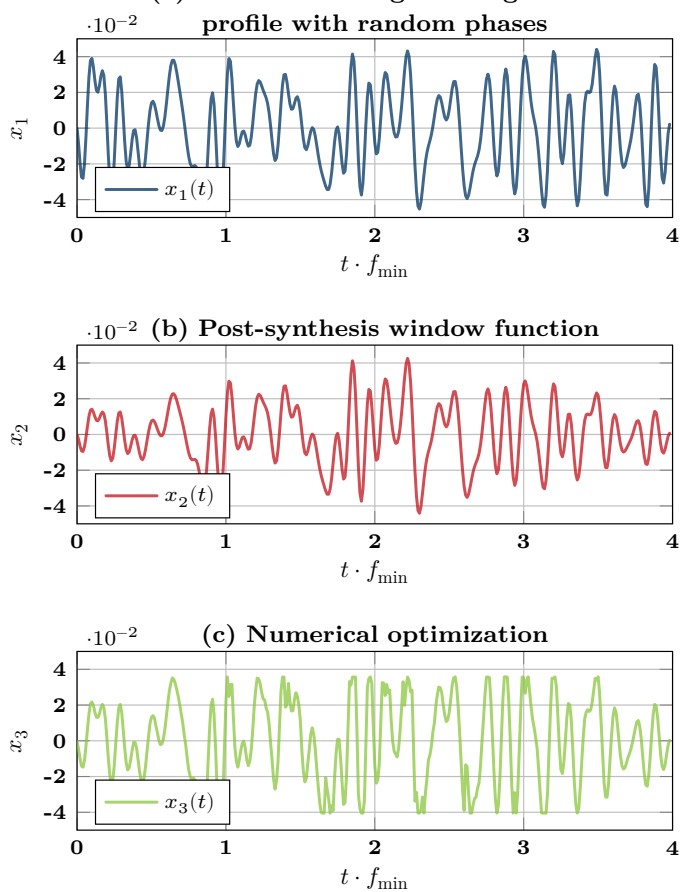

(d) Frequency domain comparison

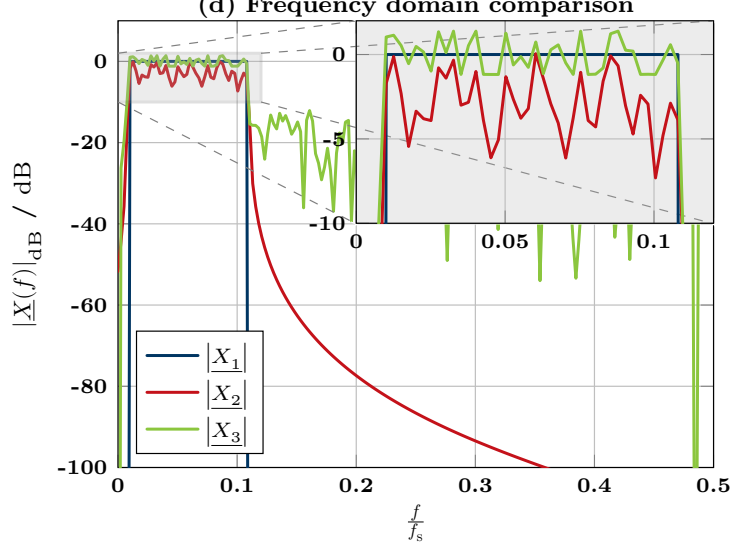

Figure 8. Time domain waveforms resulting from (a) IDFT of a rectangular magnitude profile with random phases $\left(x_{1}(t)\right)$, (b) additional post-synthesis window function multiplication $\left(x_{2}(t)\right)$ and (c) further numerical optimization including intra-synthesis window function multiplication $\left(x_{3}(t)\right)$. In (d), the spectral magnitude of all waveforms is compared.

been chosen to be $30 \mathrm{~A}$ in order to achieve a voltage response amplitude which does not violate the linearity constraint for the given cell type.

$i_{\mathrm{DUT}}(t)=\frac{\hat{i}_{\mathrm{pp}}}{\hat{x}_{3, \mathrm{pp}}} x_{3}(t)$

Both the target waveform and the measured signal are shown in time and frequency domain in Fig. 9. The synthesis con-
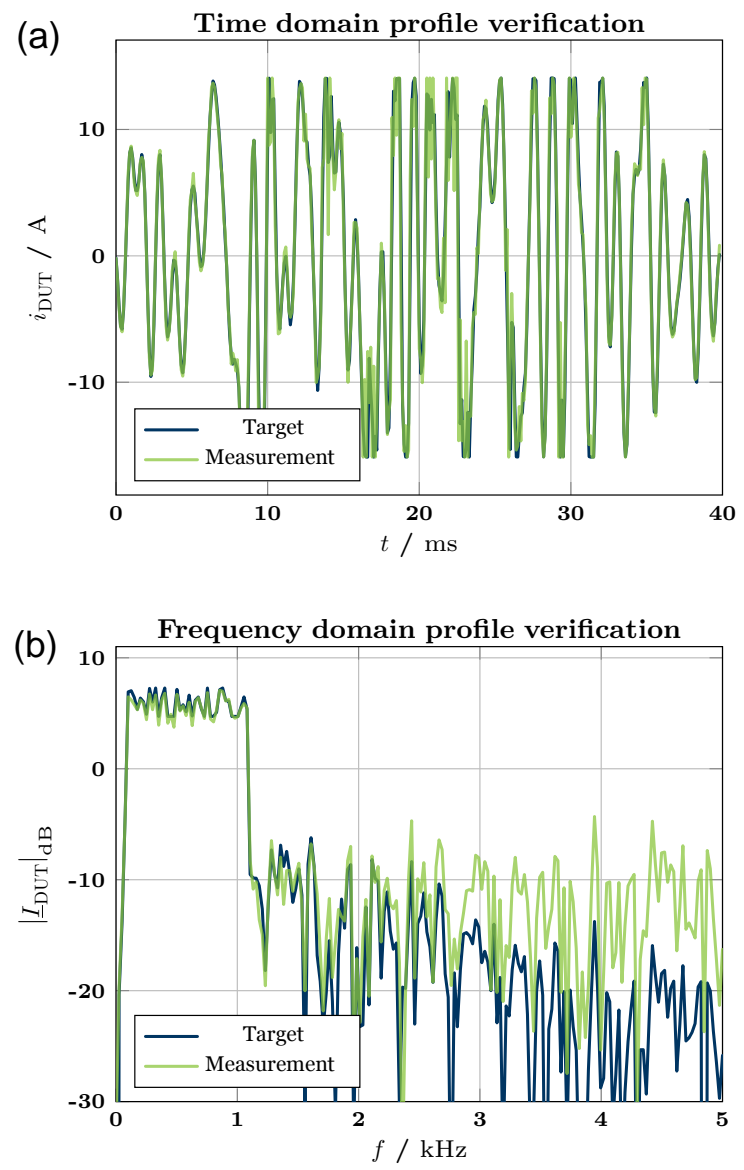

Figure 9. Verification of the time (top) and frequency domain (bottom) characteristics of the proposed waveform $x_{3}(t)$.

cept is verified by the close consistency of the target and measured signal both in time and frequency domain.

To evaluate the suitability of the proposed waveform for EIS studies, the impedance is measured in the frequency range from $10 \mathrm{mHz}$ to $1 \mathrm{kHz}$ using both the established stepped-sine approach (51 frequency points in $30 \mathrm{~min}$ ) and the proposed measurement procedure based on $x_{3}(t)$ in five different timescales requiring $8 \mathrm{~min}$ in total. The Nyquist plots of the resulting impedance spectra are depicted in Fig. 10. The results of both procedures show good concordance, indicating the suitability of the proposed time domain method.

Especially at low frequencies, the spectrum obtained from the optimized waveform is enriched by the increased frequency resolution achieved by the broadband excitation.

To evaluate the data quality and integrity of the measured spectra, a Kramers-Kronig compliance test is performed on the impedance data obtained using both methods. The Kramers-Kronig residual distribution for the impedance spectra is depicted in Fig. 11. For both spectra, a homogeneous distribution of the residual can be seen. The Kramers- 


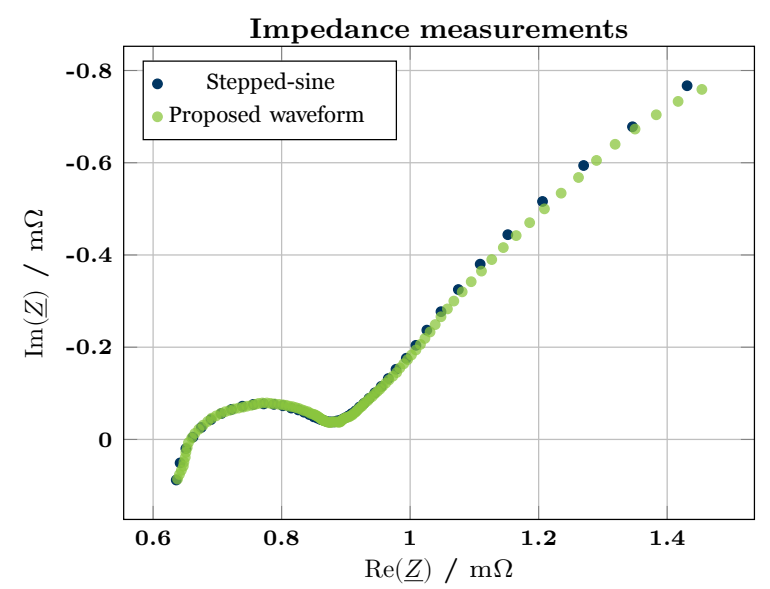

Figure 10. Impedance measured using stepped sine (blue) and $x_{3}(t)$ (green) excitation.

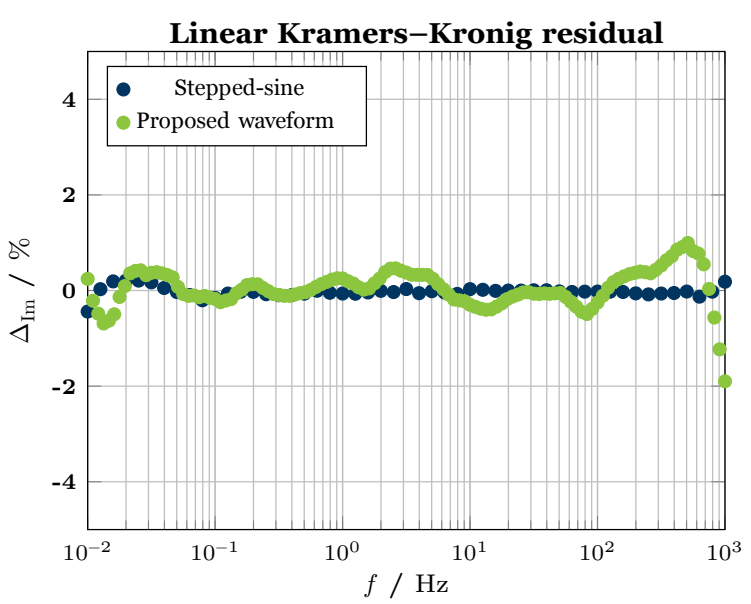

Figure 11. Linear Kramers-Kronig residual for impedance spectra measured using stepped-sine (blue) and $x_{3}(t)$ (green) excitation.

Kronig compliance of both spectra is confirmed for both of them, as no significant deviation can be recognized. The observed residual is caused by measurement noise.

Due to the increased available excitation power for each frequency in stepped-sine measurements, a lower residual is achieved compared to measurements based on the proposed waveform at the cost of substantially higher charge throughput and excessively longer measurement duration. In Table 3, a quantitative comparison of both measurement procedures is given. The table also includes results of an EIS measurement using the previously presented waveform $x_{3 b}$ (Haußmann and Melbert, 2016). While measurement duration and charge throughput are equivalent for the measurements based on $x_{3}$ and $x_{3 \mathrm{~b}}, x_{3}$ yields a slightly lower residual value, indicating enhanced data quality.
Table 3. Quantitative comparison of stepped-sine and optimized time domain EIS measurements on an automotive lithium ion cell.

\begin{tabular}{lrcc}
\hline Method & Stepped sine & $x_{3 \mathrm{~b}}(t)^{\mathrm{a}, \mathrm{b}}$ & $x_{3}(t)^{\mathrm{b}}$ \\
\hline$f_{\min }$ & $10 \mathrm{mHz}$ & $10 \mathrm{mHz}$ & $10 \mathrm{mHz}$ \\
$f_{\max }$ & $1 \mathrm{kHz}$ & $1 \mathrm{kHz}$ & $1 \mathrm{kHz}$ \\
Freq. points per decade & 10 & $24^{\mathrm{c}}(+140 \%)$ \\
Total duration & $30 \mathrm{~min}$ & $8 \mathrm{~min}(-73 \%)$ \\
Charge throughput & $2.41 \mathrm{Ah}$ & $0.27 \mathrm{Ah}(-88 \%)$ \\
Mean KK residual & $0.07 \%$ & $0.28 \%$ & $0.27 \%$
\end{tabular}

a Presented in Haußmann and Melbert (2016).

b A sequence of five pulses in different timescales is performed to investigate the entire frequency range of interest.

${ }^{c}$ Frequency resolution is chosen arbitrarily.

\section{Conclusions}

A waveform-shaping method to generate broadband time domain signals based on user-defined magnitude profiles was developed and optimized for high signal power at low peakto-peak amplitudes. Compared to common pulse waveforms with equal spectral properties, a significant decrease in peakto-peak amplitude is achieved by means of random phase variation. The signal enhancements introduced by employing window functions are inherently included in the optimization work flow. By combining statistical and numerical optimization approaches, even lower peak-to-peak amplitude values are achieved. The resulting waveform exhibits only negligible pass-band ripple. As a consequence, the overall excitation signal power in EIS can be increased significantly while ensuring quasi-linear excitation of the DUT.

The concept was verified in simulation and measurement. EIS results based on the optimized time domain waveform show good concordance with reference stepped sine EIS measurements, drastically reducing both measurement duration and charge throughput. The numerical optimization is based on a parametric cost function, which allows to emphasize the target waveform properties in accordance to application-specific constraints. Therefore, the waveform-shaping method is not restricted to the synthesis of impedance spectroscopy excitation waveforms, but can also supplement other system identification applications, including audio channel characterization and ultrasonic measurement systems.

\section{Data availability}

The dataset used in this work is available in the Supplement. 


\section{The Supplement related to this article is available online at doi:10.5194/jsss-6-65-2017-supplement.}

Competing interests. The authors declare that they have no conflict of interest.

Edited by: A. Schütze

Reviewed by: two anonymous referees

\section{References}

Banerjee, A., Ziv, B., Luski, S., Aurbach, D., and Halalay, I. C.: Increasing the durability of Li-ion batteries by means of manganese ion trapping materials with nitrogen functionalities, J. Power Sources, 341, 457-465, doi:10.1016/j.jpowsour.2016.12.036, 2017.

Barai, A., Chouchelamane, G. H., Guo, Y., McGordon, A., and Jennings, P.: A study on the impact of lithium-ion cell relaxation on electrochemical impedance spectroscopy, J. Power Sources, 280, 74-80, doi:10.1016/j.jpowsour.2015.01.097, 2015.

Fischnaller, M., Melbert, J., Scharner, S., and Eichner, T.: Test Center for Aging Analysis and Characterization of LithiumIon Batteries for Automotive Applications, in: SAE 2011 World Congress \& Exhibition, SAE Technical Paper Series, SAE International400 Commonwealth Drive, Warrendale, PA, USA, doi:10.4271/2011-01-1374, 2011.

Gamry Instruments, I.: OptiEIS: A Multisine Implementation Application Note Rev. 1.0, https://www.gamry.com/ application-notes/EIS/optieis-a-multisine-implementation/ (last access: 25 January 2017), 2011.

Guerrero Cervera, T., Vega-Leal, A. P., Adame García, G., and Brey Sánchez, J.: PEMFC impedance spectroscopy using synthetic wide-band signals, Int. J. Hydrogen Energ., 39, 40054008, doi:10.1016/j.ijhydene.2013.06.078, 2014.

Guillaume, P., Verboven, P., Vanlanduit, S., and Parloo, E.: Multisine Excitations - New Developements and Applications in Modal Analysis, in: Proceedings of IMAC-XIX: a conference on structural dynamics, vol. 4359 of SPIE proceedings series, 15431549, SPIE, Bellingham, Washington, 2001.

Hanke-Bourgeois, M.: Grundlagen der Numerischen Mathematik und des Wissenschaftlichen Rechnens, Mathematische Leitfäden, Vieweg+Teubner Verlag/GWV Fachverlage GmbH Wiesbaden, Wiesbaden, 2. überarbeitete und erweiterte Auflage edn., 149-198, doi:10.1007/978-3-8351-9020-7, 2006.

Harris, F. J.: On the use of windows for harmonic analysis with the discrete Fourier transform, Proc. IEEE, 66, 51-83, doi:10.1109/PROC.1978.10837, 1978.

Haußmann, P. and Melbert, J.: Impedance spectroscopy on lithium ion cells for automotive applications with optimized measurement duration and frequency resolution: AMA Service GmbH, Von-Münchhausen-Str. 49, 31515 Wunstorf, Germany, in: 18. GMA/ITG-Fachtagung Sensoren und Messsysteme, doi:10.5162/sensoren2016/4.4.2, 2016.
Huang, Q.-A., Shen, Y., Huang, Y., Zhang, L., and Zhang, J.: Impedance Characteristics and Diagnoses of Automotive Lithium-Ion Batteries at $7.5 \%$ to $93.0 \%$ State of Charge, Electrochim. Acta, 219, 751-765, doi:10.1016/j.electacta.2016.09.154, 2016.

Klotz, D., Schönleber, M., Schmidt, J. P., and Ivers-Tiffée, E.: New approach for the calculation of impedance spectra out of time domain data, Electrochim. Acta, 56, 8763-8769, doi:10.1016/j.electacta.2011.07.096, 2011.

Koch, R. and Jossen, A.: Speed Optimized Multisine Stimuli for Electrochemical Impedance Spectroscopy with a Switched Mode Excitation, in: 2014 IEEE Vehicle Power and Propulsion Conference (VPPC), 1-6, doi:10.1109/VPPC.2014.7007135, 2014.

Land, R., Annus, P., and Min, M.: Time-frequency impedance spectroscopy: excitation considerations, in: IMEKO TC4 International Symposium on Novelties in Electrical Measurements and Instrumentations, vol. 1 of 15th IMEKO TC4 Internatinal Symposium on Novelties in Electrical Measurements and Instrumentations Proceedings, vol. 15, 328-221, 2007.

Lohmann, N., Haussmann, P., Wesskamp, P., Melbert, J., and Musch, T.: Employing Real Automotive Driving Data for Electrochemical Impedance Spectroscopy on Lithium-Ion Cells, SAE Int. J. Alt. Power, 4, 308-317, doi:10.4271/2015-01-1187, 2015a.

Lohmann, N., Weßkamp, P., Haußmann, P., Melbert, J., and Musch, T.: Electrochemical impedance spectroscopy for lithium-ion cells: Test equipment and procedures for aging and fast characterization in time and frequency domain, J. Power Sources, 273, 613-623, doi:10.1016/j.jpowsour.2014.09.132, 2015b.

Lyons, R. G.: Understanding digital signal processing, Prentice Hall, Upper Saddle River, NJ, 3rd ed. edn., 60-73, 2011.

Møller, A. R.: Systems identification using pseudorandom noise applied to a sensorineural system, Comput. Math. Appl., 12, 803814, doi:10.1016/0898-1221(86)90065-9, 1986.

Sanchez, B., Vandersteen, G., Bragos, R., and Schoukens, J.: Optimal multisine excitation design for broadband electrical impedance spectroscopy, Measurement Sci. Technol., 22, 115601, doi:10.1088/0957-0233/22/11/115601, 2011.

Schmidt, J. P., Chrobak, T., Ender, M., Illig, J., Klotz, D., and Ivers-Tiffée, E.: Studies on LiFePO4 as cathode material using impedance spectroscopy, J. Power Sources, 196, 5342-5348, doi:10.1016/j.jpowsour.2010.09.121, 2011.

Schönleber, M. and Ivers-Tiffée, E.: Approximability of impedance spectra by RC elements and implications for impedance analysis, Electrochem. Commun., 58, 15-19, doi:10.1016/j.elecom.2015.05.018, 2015.

Sundararajan, D.: The Discrete Fourier Transform: Theory, Algorithms and Applications, World Scientific, https://books.google. de/books?id=54kTgFg5IVgC (last access: 25 January 2017), 2001.

Symons, P.: Digital waveform generation, Cambridge University Press, Cambridge, UK, http://proquest.tech.safaribooksonline. de/9781107502048 (last access: 25 January 2017), 2014.

Weßkamp, P., Haußmann, P., and Melbert, J.: 600-A Test System for Aging Analysis of Automotive Li-Ion Cells With High Resolution and Wide Bandwidth, IEEE T. Instrum. Meas., 65, 16511660, doi:10.1109/TIM.2016.2534379, 2016. 